\title{
Strain enhancement during annealing of GaAsN alloys
}

\author{
Q. D. Zhuang ${ }^{\text {a) }}$ and A. Krier \\ Physics Department, Lancaster University, Lancaster, LA1 4YB, United Kingdom \\ C. R. Stanley \\ Department of Electronics and Electrical Engineering, University of Glasgow, Glasgow, G12 8LT, United \\ Kingdom
}

(Received 28 November 2006; accepted 8 February 2007; published online 31 May 2007)

\begin{abstract}
We have investigated the effect of rapid thermal annealing (RTA) on the optical and structural properties of GaAsN alloys using photoluminescence (PL) and double-crystal x-ray diffraction. We observe a significant anomalous strain enhancement during RTA and a blueshift of the PL peak energy accompanied by a reduction in the emission linewidth. The PL features are attributed to an improvement in the homogeneity of the alloy, and the strain enhancement reflects a change in $\mathrm{N}$-related complexes during annealing. Based on a defect model, an interstitial nitrogen concentration of $1.8 \times 10^{19} \mathrm{~cm}^{-3}$ is deduced prior to annealing. (C) 2007 American Institute of Physics. [DOI: 10.1063/1.2717603]
\end{abstract}

The III-V dilute nitrides have interesting physical properties and considerable potential for applications in optical fiber communications in the $1.3-1.5 \mu \mathrm{m}$ spectral range ${ }^{1}$ as well as for mid-infrared optoelectronics. ${ }^{2}$ It is well known that the incorporation of small concentrations of nitrogen into GaAs reduces the band gap energy dramatically due to the formation of spatially separated and sharply localized band edge states around the $\mathrm{N}$ atoms. ${ }^{3}$ However, the material quality degrades severely with increasing nitrogen composition, especially with respect to the photoluminescence (PL) emission efficiency. ${ }^{4}$ It is widely reported that postgrowth thermal treatments, such as rapid thermal annealing (RTA) and oven annealing, can improve the PL emission efficiency of both GaAsN bulk ${ }^{5,6}$ and $\mathrm{Ga}(\mathrm{In}) \mathrm{AsN}$ quantum wells $(\mathrm{QWs}){ }^{7-9}$ Such improvements are commonly attributed to the annihilation of competing nonradiative point defects. However, the identification of these point defects remains unclear, although vacancies, ${ }^{9} \mathrm{~N}-\mathrm{H}$ complexes, ${ }^{5}$ and nitrogen interstitials ${ }^{8}$ have been considered to be nonradiative recombination centers. Most of the RTA studies to date have concentrated on structures containing dilute nitride quantum wells. However, the intermixing of group IIIs has a dramatic effect on PL spectra and x-ray rocking curves, and leads to difficulties in understanding unambiguously the true effects of postgrowth annealing on the structural properties and point defects. The annealing of dilute nitride bulk materials could be an effective way to investigate the structural changes occurring during the annealing process, thus helping to reveal the nature of the point defects in these as-grown materials. Bosker et al. ${ }^{10}$ have reported a N "kick-off" diffusion mechanism involving interstitial $\mathrm{N}$ and substitutional $\mathrm{N}$ exchange during the annealing of GaAsN, but there have been very few fundamental studies reported to date. ${ }^{5,6,10,11}$ In this article, we report on the effect of RTA of GaAsN bulk alloys studied using PL and double-crystal x-ray diffraction (DCXRD) measurements. Our investigation reveals a blue-

${ }^{a)}$ Electronic mail: q.zhuang@lancaster.ac.uk shift of PL emission peak energy and a decrease of the emission linewidth after RTA. More importantly, we observed a significant anomalous strain enhancement during annealing which reflects the changes in N-related point defects.

The GaAsN epilayers were grown by solid source molecular beam epitaxy (MBE) using a radio frequency (rf) plasma nitrogen source. GaAsN epilayers, $200 \mathrm{~nm}$ thick, were deposited after the growth of a GaAs buffer layer at $480{ }^{\circ} \mathrm{C}$, using a growth rate of $1.0 \mu \mathrm{m} / \mathrm{h}$ and a rf power of $200 \mathrm{~W}$. The nitrogen background was set at $5.0 \times 10^{-7}$ Torr. The reflected high-energy electron diffraction (RHEED) pattern was clearly $(2 \times 4)$ for all of the growths. Samples 4 $\times 4 \mathrm{~mm}^{2}$ were annealed between $600{ }^{\circ} \mathrm{C}$ and $900{ }^{\circ} \mathrm{C}$ for 60 $\mathrm{s}$ using a RTA system with a $\mathrm{N}_{2}$ ambient. The samples were covered by a GaAs wafer (face to face) to prevent the loss of arsenic at elevated temperatures. PL and DCXRD were measured on each piece of the sample before and after annealing, and were compared to investigate the effect of annealing.

Figure 1(a) shows typical $10 \mathrm{~K}$ PL spectra from GaAsN before and after annealing at $750{ }^{\circ} \mathrm{C}$ for $60 \mathrm{~s}$. The emission peak at $\sim 1.02 \mu \mathrm{m}$ originates from dilute nitride. The broad band near $1.5 \mu \mathrm{m}$ might originate from recombination via defect levels associated with oxygen. ${ }^{12}$ After annealing, a blueshift of the PL peak energy and a reduction of the linewidth are clearly evident. However, the normally reported improvement in integrated PL intensity is not observed. This is not understood clearly, but presumably is due to surface degradation and an increased recombination velocity after annealing. ${ }^{6}$

Figure 1(b) shows the dependence of PL peak energy and linewidth narrowing on annealing temperature. The blueshift increases with increasing annealing temperature and saturates above $750{ }^{\circ} \mathrm{C}$, while the linewidth decreases and reaches a minimum between $750{ }^{\circ} \mathrm{C}$ and $800{ }^{\circ} \mathrm{C}$. The two most likely contributions to the blueshift of emission peak energy are (i) loss of nitrogen, due to the correlation of nitrogen composition with the peak energy of PL emission at low temperature ${ }^{7}$ and (ii) an improvement in uniformity of 

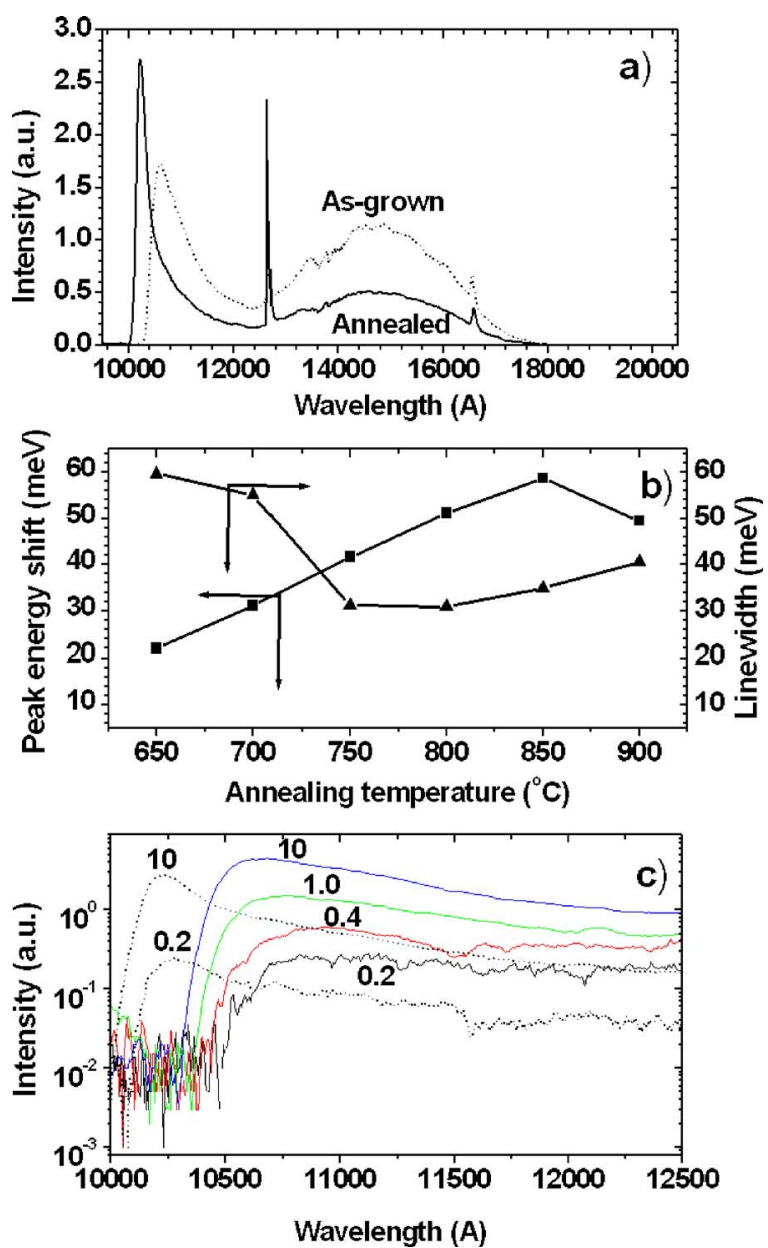

FIG. 1. (a) $10 \mathrm{~K}$ PL spectra from as-grown GaAsN and samples annealed at $750{ }^{\circ} \mathrm{C}$ for $60 \mathrm{~s}$ (peaks at $\sim 1.27$ and $1.56 \mu \mathrm{m}$ are high orders from the laser); (b) PL peak energy shift and linewidth changes with increasing annealing temperature; (c) $10 \mathrm{~K} \mathrm{PL}$ spectra at different laser exciting powers (from 0.2 to $10 \mathrm{~mW}$ ) for the as-grown sample (dotted line) and the annealed sample (solid line).

the nitrogen distribution. ${ }^{9}$ Even though a loss of nitrogen after annealing has been reported, ${ }^{11}$ this is unlikely in our experiments due to the strong Ga-N bond and short annealing time. More importantly, no corresponding decrease of the splitting angle in the DXRD measurements after annealing was observed. We therefore attribute the blueshift to an improvement in the homogeneity of the alloy. Previous studies indicate that the low-temperature PL emission originates from the recombination of localized excitons trapped by N-related states near the GaAsN band edge due to the nonuniformity of the nitrogen distribution. ${ }^{13}$ The PL spectrum exhibits the features of a very asymmetric line shape with a sharp, high-energy cutoff and an exponential low-energy tail. This transition energy is lower than the band-band energy and the emission correlates as expected with laser excitation power and temperature. ${ }^{14}$ Figure 1 (c) shows the $4 \mathrm{~K}$ PL spectra measured at laser powers in the range of $0.5-10 \mathrm{~mW}$ for as-grown material and a sample annealed at $750{ }^{\circ} \mathrm{C}$. A blueshift of $27 \mathrm{meV}$ was observed for as-grown sample and only $5 \mathrm{meV}$ for the annealed sample. The reduction in the blueshift is attributed to an improvement of the uniformity of the nitrogen distribution, which decreases the localization energy, and as a consequence the transition energy moves

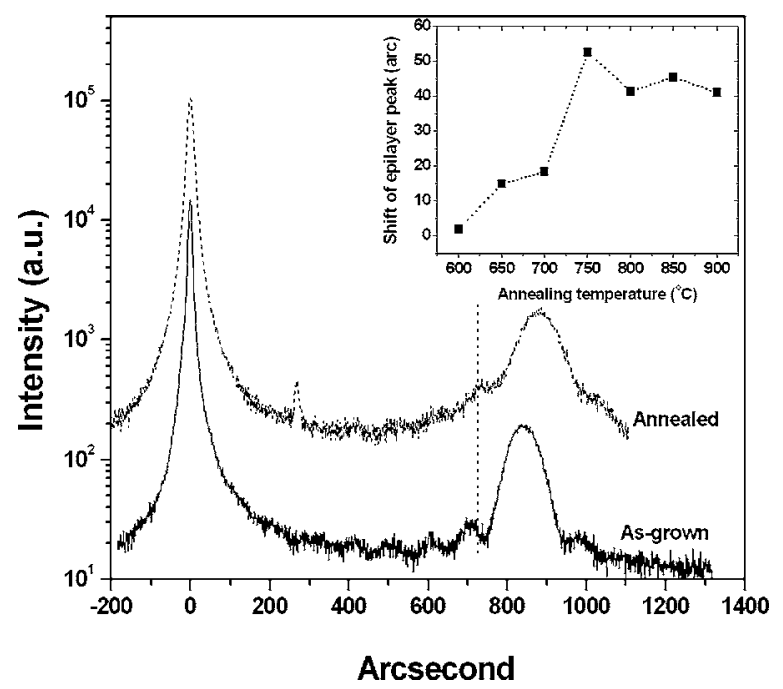

FIG. 2. X-ray rocking curves from samples of as-grown GaAsN and after annealing at $800{ }^{\circ} \mathrm{C}$ for $60 \mathrm{~s}$ (the central sharp peak in the annealed sample is from the GaAs wafer used as a carrier for the x-ray measurements). Inset shows the shift of the rocking curve angle with annealing temperature.

closer to the band gap (i.e., blueshift at elevated annealing temperature). This improvement is reflected in narrowing of the linewidth.

DCXD rocking curves of as-grown and annealed samples (at $800{ }^{\circ} \mathrm{C}$ ) are shown in Fig. 2. An anomalous but significant strain enhancement is observed after annealing. The inset shows the strain enhancement increases with increasing annealing temperature and saturates above $750{ }^{\circ} \mathrm{C}$. The enhanced strain always implies a high composition of substitutional nitrogen. Similar strain enhancement has been reported for GaAsN alloys oven-annealed at $450{ }^{\circ} \mathrm{C}$ for 5 $\mathrm{min}$, and was attributed to increased $\mathrm{N}$ incorporation during annealing. ${ }^{6}$ However, this is unlikely in our experiments due to the absence of a corresponding redshift of PL peak energy and the short annealing time. We believe the strain enhancement might be attributed to N-As split interstitials.

Although it is believed that nitrogen incorporates in GaAs predominantly on the As sublattice, there are some other $\mathrm{N}$ configurations such as (i) N-N split interstitials; (ii) $\mathrm{N}$-As split interstitials; and (iii) the isolated interstitial N. It has been reported that the isolated interstitial $\mathrm{N}$ is unlikely to be formed due to a high formation energy. ${ }^{15}$ Veal et al. ${ }^{16}$ reported that no interstitial exists in GaAsN; this might be due to the different growth conditions. It is reported that the $(2 \times 1)$ reconstruction could suppress interstitial nitrogen significantly. ${ }^{17}$ Typically, a large fraction of interstitial nitrogen was revealed by nuclear reaction analysis and Rutherford backscattering spectrometry ${ }^{17,18}$ and by comparison of data

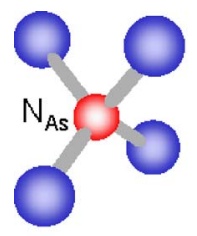

(a)

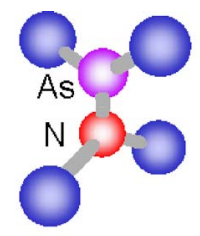

(b)

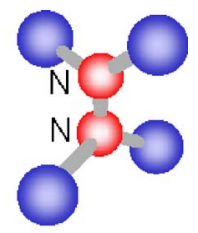

(c)
FIG. 3. Possible configurations of nitrogen in GaAsN; (a) substitutional nitrogen- $\mathrm{N}_{\mathrm{As}}$; (b) split interstitial-As-N; (c) split interstitial-N-N. 


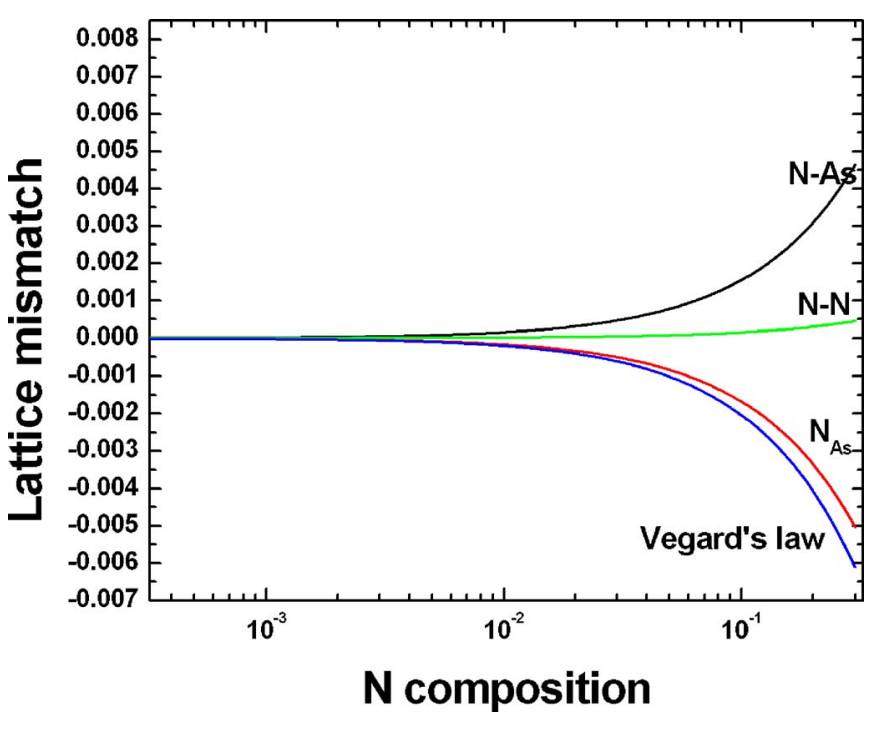

FIG. 4. Lattice mismatch of GaAsN/GaAs caused by substitutional $\mathrm{N}_{\mathrm{As}}$ split interstitial complexes of As- $\mathrm{N}$ and $\mathrm{N}-\mathrm{N}$, and their dependence on $\mathrm{N}$ content.

from x-ray and secondary ion mass spectrometry. ${ }^{15,19}$ Figure 3 displays the three possible $\mathrm{N}$-related complexes. The effect of these N-related complexes on the lattice constant can be calculated based on a simple model produced by Chen $e t$ $a l .{ }^{20}$ Equations (1) and (2) give the lattice strain $\Delta a / a$ caused by $\mathrm{N}_{\mathrm{As}}$, substitutional and N-As or N-N split interstitial complexes, respectively,

$$
\begin{aligned}
& \frac{\Delta a}{a}=\frac{C_{11}+2 C_{12}}{C_{11}} \frac{r_{\mathrm{N}}-r_{\mathrm{As}}}{r_{\mathrm{Ga}}+r_{\mathrm{As}}} \frac{x}{2}, \\
& \frac{\Delta a}{a}=\frac{C_{11}+2 C_{12}}{C_{11}} \frac{d_{b}-r_{\mathrm{Ga}}-r_{\mathrm{As}}}{r_{\mathrm{Ga}}+r_{\mathrm{As}}} \frac{x}{2} .
\end{aligned}
$$

Here, $r_{\mathrm{N}}, r_{\mathrm{As}}$, and $r_{\mathrm{Ga}}$ are the covalent radii of $\mathrm{N}, \mathrm{As}$, and $\mathrm{Ga}$, respectively. $C_{11}, C_{12}$ are the stiffness coefficients of the crystals. $d_{b}=\sqrt{3} r_{\text {eff }} / 3+\sqrt{\left(r_{\text {eff }}+r_{\mathrm{Ga}}\right)^{2}-2 r_{\text {eff }}^{2} / 3}$ is the distance of the N-As complex from its nearest neighbors; $r_{\text {eff }}=\left(r_{\mathrm{N}}\right.$ $\left.+r_{\mathrm{As}}\right) / 2$ is the effective bond radius.

The effects of substitutional $\mathrm{N}_{\mathrm{As}}$, split interstitial N-As, and split interstitial N-N complexes on the mismatch of GaAsN epilayers at various $\mathrm{N}$ contents with respect to the GaAs substrate are shown in Fig. 4. The effect of N-N split interstitials can be neglected since there is no contribution to strain enhancement. Figure 4 also shows clearly that contrary to substitutional nitrogen, which gives rise to a reduction in lattice constant, the N-As split interstitial dilates the lattice constant. Hence, its existence compensates the tensile strain of the GaAsN alloy. During annealing, the N-As split interstitials become broken to form Ga-As bonds, and the nitrogen is thus released and diffuses out. The annihilation of the split interstitials decreases the dilation of the lattice constant and results in a strain enhancement. It can be expected that by increasing the annealing temperature more interstitial bonds will be broken, with further outdiffusion of $\mathrm{N}$ and a corresponding shift in the DCXRD curve, which would become constant once the split interstitials are completely an- nihilated. The strain enhancement therefore provides the possibility of determining the concentration of $\mathrm{N}$-As split interstitials.

Above a temperature of $750{ }^{\circ} \mathrm{C}$, the DCXRD angle does not change and we may assume that nearly all the interstitials have been annihilated. Consequently, the x-ray result on the annealed sample gives the concentration of substitutional nitrogen, which is $1.78 \%$. However, due to the existence of interstitial nitrogen the $\mathrm{x}$-ray result on as-grown sample leads to an apparent nitrogen composition of $1.7 \%$. We believe the difference is caused by the annihilation of interstitial nitrogen, and the concentration of interstitial nitrogen can thereby be deduced from this difference by simulating the contributions of interstitial nitrogen to the lattice constant (as shown in Fig. 4). We estimate that the concentration of interstitial nitrogen in our sample is $1.8 \times 10^{19} \mathrm{~cm}^{-3}$, in excellent agreement with a recent report ${ }^{18}$ of $2 \times 10^{19} \mathrm{~cm}^{-3}$. To determine the total nitrogen composition (substitutional and interstitial nitrogen) of the as-grown GaAsN, this interstitial nitrogen has to be included, giving a total nitrogen composition of $1.84 \%$.

In summary, we have studied the effect of RTA on the optical and structural properties of bulk GaAsN alloys using low-temperature PL spectroscopy and x-ray diffraction measurements. With increasing annealing temperature, a blueshift of PL emission peak energy and narrowing of the emission linewidth have been observed. DCXRD reveals a significant strain enhancement as a result of RTA. This can be attributed to the annihilation of As-N split interstitials formed during MBE growth. Based on a point defect model, we have deduced the concentration of interstitial nitrogen to be $1.8 \times 10^{19} \mathrm{~cm}^{-3}$, and the composition of substitutional nitrogen to be $1.78 \%$.

The research was support by the Engineering and Physical Sciences Research Council (U.K.) through the PHOTON project.

${ }^{1}$ M. Kondow, K. Uomi, K. Hosomi, and T. Mozume, Jpn. J. Appl. Phys., Part 2 33, L1056 (1994).

${ }^{2}$ D. K. Shih, H. H. Lin, and Y. H. Lin, Electron. Lett. 37, 1342 (2001).

${ }^{3}$ W. Shan, W. Walukiewicz, J. W.Ager III, E. E. Haller, J. F. Geisz, D. J. Friedman, J. M. Olson, S. R. Kurtz et al., Phys. Rev. Lett. 82, 1221 (1999).

${ }^{4}$ H. P. Xin and C. W. Tu, Appl. Phys. Lett. 72, 2442 (1998).

${ }^{5}$ E. V. Rao, A. Ougazzaden, Y. Le Bellego, and M. Juhel, Appl. Phys. Lett. 72, 1409 (1998)

${ }^{6}$ S. Francoeur, G. Sivaraman, Y. Qiu, S. Nikishin, and H. Temkin, Appl. Phys. Lett. 72, 1857 (1998).

${ }^{7}$ I. A. Buyanova, G. Pozina, P. N. Hai, N. Q. Thinh, J. P. Bergman, W. M. Chen, H. P. Xin, and C. T. Tu, Appl. Phys. Lett. 77, 2325 (2000).

${ }^{8}$ W. Li, M. Pessa, T. Ahlgren, andJ. Decker, Appl. Phys. Lett. 79, 1094 (2001).

${ }^{9}$ E. Tournié, M. A. Pinault, and A. Guzman, Appl. Phys. Lett. 80, 4148 (2002).

${ }^{10}$ G. Bosker, N. Stolwijk, J. V. Thordson, U. Sodervall, and T. G. Andersson, Phys. Rev. Lett. 81, 3443 (1998).

${ }^{11}$ S. Gwo, S.-Y. Huang, and T. R. Yang, Phys. Rev. B 64, 113312 (2001).

${ }^{12}$ A. R. Calawa, Appl. Phys. Lett. 38, 701 (1981).

${ }^{13}$ I. A. Buyanova, W. M. Chen, G. Pozina, J. P. Bergman, B. Monemar, H. P. Xin, and C. W. Tu, Appl. Phys. Lett. 75, 501 (1999).

${ }^{14}$ A. Polimeni, M. Capizzi, M. Geddo, M. Fischer, M. Reinhardt, and A. Forchel, Phys. Rev. B 63, 195320 (2001). 
${ }^{15}$ W. Li, M. Pessa, and J. Likonen, Appl. Phys. Lett. 78, 2864 (2001).

${ }^{16}$ T. D. Veal, I. Mahboob, L. F. J. Piper, C. F. McConville, and M. Hopkinson, Appl. Phys. Lett. 85, 1550 (2004).

${ }^{17}$ M. Reason, H. A. McKay, W. Ye, S. Hanson, R. S. Goldman, and V. Rotberg, Appl. Phys. Lett. 85, 1692 (2004).
${ }^{18}$ T. Ahlgren, E. Vainonen-Ahlgren, J. Likonen, W. Li, and M. Pessa, Appl. Phys. Lett. 80, 2314 (2002).

${ }^{19}$ W. J. Fan, S. F. Yoon, T. K. Ng, S. Z. Wang, W. K. Loke, R. Liu, and A. Wee, Appl. Phys. Lett. 80, 4136 (2002).

${ }^{20}$ N. F. Chen, Y. Wang, H. He, and L. Lin., Phys. Rev. B 54, 8516 (1996). 